\title{
Optimization of Excitation Magnitudes and Phases for Maximum Efficiencies in a MISO Wireless Power Transfer System
}

\author{
Hyeongwook Lee $\cdot$ Seunghyun Boo $\cdot$ Gunyoung Kim • Bomson Lee*
}

\begin{abstract}
This paper presents a method for solving receiver misalignment (axial or angular) problems in wireless power transfer systems using a multiple-input single-output system. The optimum magnitudes and phases of the transmitter voltages and receiver load for maximum efficiency are derived in convenient analytical forms when negligible mutual couplings between transmitters. These solutions are validated by genetic algorithm optimization and electromagnetic-simulation results for a design ex-ample of two transmitters and one rotating receiver.
\end{abstract}

Key Words: Maximum Efficiency, Misalignment, Optimum Input Voltage, Optimum load, Wireless Power Transfer.

\section{INTRODUCTION}

Wireless power transfer (WPT) using resonance through magnetic couplings has been actively studied since it was proposed by Soljacic in 2007 [1]. Efficiency analysis of a onetransmitter $(\mathrm{Tx})$ and one-receiver $(\mathrm{Rx})$ system facing each other has been sufficiently performed, and related products can be found in the market. However, the degree of freedom of the receiver positions and postures is still limited. Some studies have been conducted to solve the misalignment problems occurring between $\mathrm{Tx}$ and $\mathrm{Rx}$. The misalignment can be divided into two types: axial and angular. A theoretical research on multipleinput single-output (MISO) systems was conducted in [2]. In [3], an energy-efficient and adaptive design method for wireless power transfer in electric vehicles was proposed to alleviate the axial misalignment problem using multiple transmitters. To maximize the power transfer efficiency, the $\mathrm{Tx}$ phases and $\mathrm{Rx}$ loads were simultaneously optimized based on the same $\mathrm{Tx}$ magnitudes. In [4], the solution for the $\mathrm{Tx}$ voltages was presented assuming a fixed Rx load. In [5], a plurality of repeaters was attached to the Tx and $\mathrm{Rx}$ to solve the misalignment problem. In [6], the three $T x$ sources with phase differences were used to stably transfer power to an Rx. Through the phase control of orthogonally arranged Tx's, the direction of the magnetic field was controlled to solve the angular problem $[7,8]$. The solutions for the excitation voltage magnitudes and phases for a two-Tx MISO system were numerically found to maximize the efficiency [9].

In this paper, we propose a more general MISO WPT system, the excitation magnitudes and phases and the Rx load of which are simultaneously optimized to achieve maximum efficiencies despite these misalignments. Besides, we obtain the

Manuscript received July 16, 2019 ; Revised August 21, 2019 ; Accepted September 19, 2019. (ID No. 20190716-056J)

Department of Electronic Engineering, Kyung Hee University, Yongin, Korea.

"Corresponding Author: Bomson Lee (e-mail: bomson@khu.ac.kr)

This is an Open-Access article distributed under the terms of the Creative Commons Attribution Non-Commercial License (http://creativecommons.org/licenses/by-nc/4.0) which permits unrestricted non-commercial use, distribution, and reproduction in any medium, provided the original work is properly cited.

(c) Copyright The Korean Institute of Electromagnetic Engineering and Science. All Rights Reserved. 
closed-form solution of the excitation voltages for the case of negligible mutual couplings between the Tx's. In Section II, we briefly summarize the formulas for single-input single-output (SISO) systems and describe the typical two situations in which the power transfer efficiencies become zero. In Section III, based on the derived analytic solution of a two-Tx example, we deduce the solutions of multiple Tx excitation magnitudes and phases for maximum efficiency in a general MISO system. They are validated with the results based on a generic algorithm (GA) and electromagnetic (EM) simulations. The paper is concluded in Section IV.

\section{OPTIMIZATION OF A MISO SYSTEM FOR}

\section{MAXIMUM EFFICIENCY}

A SISO system is briefly reviewed to develop formulations for MISO systems. Fig. 1 shows the SISO systems in which axial and angular misalignments occur. In Fig. $1, k$ is the coupling coefficient $[10,11]$. A positive $k$ means the same magnetic flux direction crossing the Tx and Rx loops. A negative $k$ means that the magnetic flux originating from the Tx loop flows into the $\mathrm{Rx}$ loop in an opposite direction. A circle is located above the Tx loop where $k$ is zero or very small. Based on the equivalent circuit analysis in [11], efficiency $(\eta)$ can be expressed as

$$
\eta=\frac{P_{L}}{P_{i n}}=\frac{F^{2}}{\left(1+b \sqrt{1+F^{2}}\right)\left(1+\frac{1}{b} \sqrt{1+F^{2}}\right)}=\frac{\left|S_{21}\right|^{2}}{1-\left|S_{11}\right|^{2}} .
$$

In (1), $P_{\text {in }}$ is the total input power, $P_{L}$ is the power supplied to the load with $R_{L}$, and $b$ is the normalized load resistance defined

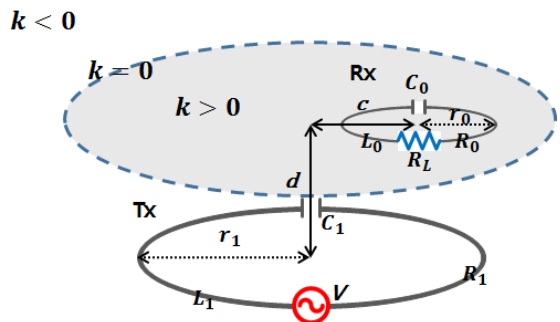

(a)

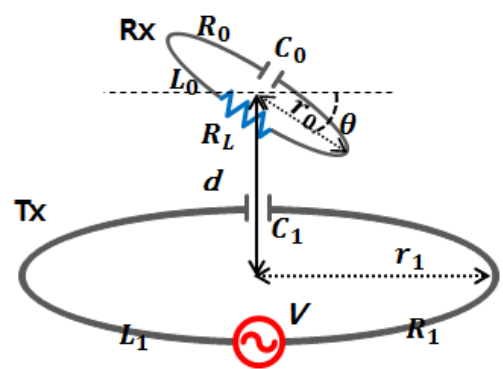

(b)

Fig. 1. Misalignment problem in a SISO system: (a) axial misalignment and (b) angular misalignment.

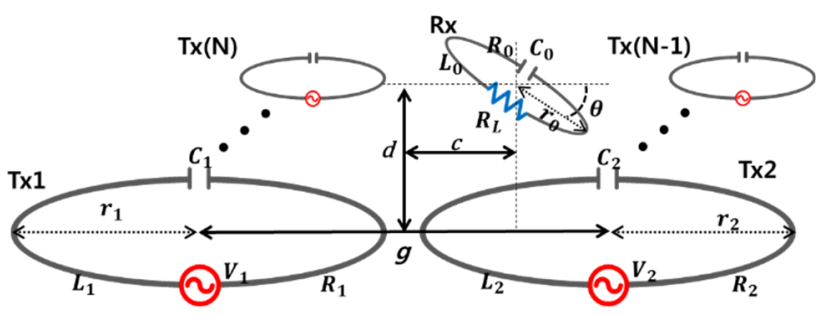

Fig. 2. MISO system with multiple transmitters and one receiver.

by $b=R_{L} / R_{L, \mathrm{opt}}$, where $R_{L, \mathrm{opt}}=R_{0} \sqrt{1+F^{2}}$ and $F$ is the figure of merit given by $F=k \sqrt{Q_{1} Q_{2}}$. It is obvious that when $b=1$, the efficiency touches an upper bound (maximum). When $b>1$ or $b<1$, the system becomes under-coupled or over-coupled, respectively, and the efficiencies fall lower than the maximum.

Fig. 2 shows a general MISO system with one Rx loop. The $\mathrm{Rx}$ loop is randomly oriented with a tilted angle $\theta$ as shown. For the MISO WPT system in Fig. 2, a circuit equation can be written as

$$
\left[\begin{array}{c}
0 \\
V_{1} \\
V_{2} \\
\vdots \\
V_{N}
\end{array}\right]=\left[\begin{array}{c}
0 \\
\left|V_{1}\right| e^{j \phi_{1}} \\
\left|V_{2}\right| e^{j \phi_{2}} \\
\vdots \\
\left|V_{N}\right| e^{j \phi_{N}}
\end{array}\right]=\left[\begin{array}{ccccc}
Z_{00} & Z_{01} & Z_{02} & \cdots & Z_{0 N} \\
Z_{10} & \ddots & & & Z_{1 N} \\
Z_{20} & & \ddots & & Z_{2 N} \\
\vdots & & & \ddots & \vdots \\
Z_{N 0} & Z_{N 1} & Z_{N 2} & \cdots & Z_{N N}
\end{array}\right]\left[\begin{array}{c}
I_{0} \\
I_{1} \\
I_{2} \\
\vdots \\
I_{N}
\end{array}\right]
$$

where the principal diagonal elements of the $Z$ matrix at the resonant design frequency are

$$
Z_{00}=R_{0}+R_{L}
$$

and

$$
Z_{i i}=R_{i}(i=1,2,3, \cdots, N) .
$$

In (3) and (4), $R_{i}^{\prime}$ s are the loss resistances of the loops, and $R_{L}$ is the load resistance of the receiver. The remaining elements of $[Z]$, mutual impedances, are given by

$$
\begin{aligned}
Z_{i j} & =-j \omega_{0} k_{i j} \sqrt{L_{i} L_{j}} \\
& =-j \omega_{0} M_{i j}(i \neq j \text { for } i, j=0,1,2, \cdots, N)
\end{aligned}
$$

where $k_{i j}$ is the coupling coefficient, $L_{i}$ is the self-inductance of the loops (or coils), and $M_{i j}$ is the mutual inductance. The column matrix $[I]$ can be obtained by $[Z]^{-1}[V]$. The total input power and the received power at the load resistance are

$$
P_{i n}=\sum_{i=1}^{N} \frac{1}{2} \operatorname{Re}\left[V_{i} I_{i}^{*}\right]
$$

and

$$
P_{L}=\frac{1}{2}\left|I_{0}\right|^{2} R_{L},
$$

respectively. Efficiency is expressed as the ratio of (6) and (7): 


$$
\eta=\frac{P_{L}}{P_{i n}}=\frac{\frac{1}{2}\left|I_{0}\right|^{2} R_{L}}{\sum_{i=1}^{N} \frac{1}{2} \operatorname{Re}\left[V_{i} I_{i}^{*}\right]}
$$

To maximize the efficiency (8), we have to optimize the $\mathrm{T} x$ voltage $\left(V_{1}, V_{2}, \ldots, V_{N}\right)$ magnitudes and phases and $R_{\mathrm{L}}$. Finding their analytical solutions considering all the system details given in (2) is usually difficult. Thus, some GA techniques, which are not easily available in industries, must be used to maximize the system efficiency in practice. However, in the case of small mutual impedances or inductances between Tx's, some convenient solutions for a maximum efficiency can be found.

We start with a simple two-Tx system $(N=2)$ under the assumption of a negligible coupling between them. For this system, the current flowing on the $\mathrm{Rx}$ loop can be analytically obtained as

$$
I_{0}=\frac{j\left(F_{1} V_{1} \sqrt{R_{2}}+F_{2} V_{2} \sqrt{R_{1}}\right)}{\gamma \sqrt{R_{0} R_{1} R_{2}}},
$$

where $F_{i}=k_{i 0} \sqrt{Q_{i} Q_{0}}, \gamma=1+F_{1}^{2}+F_{2}^{2}+\beta$, and $\beta=R_{L} / R_{0}$. The current flowing on each $\mathrm{Tx}$ loop at the resonant freuency can be expressed as

$$
I_{1}=\frac{1}{R_{1}}\left(V_{1}+j \omega_{0} M_{1} I_{0}\right)=\frac{V_{1}}{R_{1}}-\frac{F_{1}\left(F_{1} V_{1} \sqrt{R_{2}}+F_{2} V_{2} \sqrt{R_{1}}\right)}{\gamma R_{1} \sqrt{R_{2}}}
$$

and

$$
I_{2}=\frac{1}{R_{2}}\left(V_{2}+j \omega_{0} M_{2} I_{0}\right)=\frac{V_{2}}{R_{2}}-\frac{F_{2}\left(F_{2} V_{2} \sqrt{R_{1}}+F_{1} V_{1} \sqrt{R_{2}}\right)}{\gamma R_{2} \sqrt{R_{1}}},
$$

where $M_{i}\left(=k_{i 0} \sqrt{L_{i} L_{0}}\right)$ is the mutual inductance. The input power of each $\mathrm{Tx}$ is obtained as

$$
\begin{aligned}
P_{i n 1} & =\frac{1}{2} \operatorname{Re}\left[V_{1} I_{1}^{*}\right] \\
& =\frac{1}{2}\left[\frac{\left|V_{1}\right|^{2}}{R_{1}}-\frac{F_{1}^{2}\left|V_{1}\right|^{2}}{\gamma R_{1}}-\frac{F_{1} F_{2}\left|V_{1}\right|\left|V_{2}\right| \cos (\phi)}{\gamma \sqrt{R_{1} R_{2}}}\right]
\end{aligned}
$$

and

$$
\begin{aligned}
P_{i n 2} & =\frac{1}{2} \operatorname{Re}\left[V_{2} I_{2}^{*}\right] \\
& =\frac{1}{2}\left[\frac{\left|V_{2}\right|^{2}}{R_{2}}-\frac{F_{2}^{2}\left|V_{2}\right|^{2}}{\gamma R_{2}}-\frac{F_{1} F_{2}\left|V_{1}\right|\left|V_{2}\right| \cos (\phi)}{\gamma \sqrt{R_{1} R_{2}}}\right]
\end{aligned}
$$

where $\phi$ is the difference in the excitation phase $\left(\phi_{1}-\phi_{2}\right)$. The total input power and received power are given by

$$
\begin{aligned}
P_{i n} & =P_{i n 1}+P_{i n 2} \\
& =\frac{1}{2}\left[\frac{\left|V_{1}\right|^{2}}{R_{1}}+\frac{\left|V_{2}\right|^{2}}{R_{2}}-\frac{F_{1}^{2}\left|V_{1}\right|^{2}}{\gamma R_{1}}-\frac{F_{2}^{2}\left|V_{2}\right|^{2}}{\gamma R_{2}}-\frac{2 F_{1} F_{2}\left|V_{1}\right|\left|V_{2}\right| \cos (\phi)}{\gamma \sqrt{R_{1} R_{2}}}\right]
\end{aligned}
$$

and

$$
\begin{aligned}
P_{L} & =\frac{1}{2}\left|I_{0}\right|^{2} R_{L} \\
& =\frac{\beta}{2 \gamma^{2} R_{1} R_{2}}\left[F_{1}^{2}\left|V_{1}\right|^{2} R_{2}+F_{2}^{2}\left|V_{2}\right|^{2} R_{1}+2 F_{1} F_{2} \sqrt{R_{1} R_{2}}\left|V_{1}\right|\left|V_{2}\right| \cos (\phi)\right]
\end{aligned}
$$

respectively. Now, efficiency is expressed as

$$
\begin{aligned}
& \eta=\frac{P_{L}}{P_{i n}} \\
& =\frac{\beta}{\gamma} \frac{F_{1}^{2} R_{2}\left|V_{1}\right|^{2}+F_{2}^{2} R_{1}\left|V_{2}\right|^{2}+2 F_{1} F_{2} \sqrt{R_{1} R_{2}}\left|V_{1}\right|\left|V_{2}\right| \cos (\phi)}{\gamma R_{2}\left|V_{1}\right|^{2}+\gamma R_{1}\left|V_{2}\right|^{2}-F_{1}^{2} R_{2}\left|V_{1}\right|^{2}-F_{2}^{2} R_{1}\left|V_{2}\right|^{2}-2 F_{1} F_{2} \sqrt{R_{1} R_{2}}\left|V_{1}\right|\left|V_{2}\right| \cos (\phi)} .
\end{aligned}
$$

Defining $m=\left|V_{2} / V_{1}\right|$, efficiency (16) is clearly a function of three variables $(m, \phi$, and $\beta)$ given by

$$
\eta(m, \phi, \beta)=\frac{\beta}{\gamma} \frac{F_{1}^{2} R_{2}+F_{2}^{2} R_{1} m^{2}+2 F_{1} F_{2} \sqrt{R_{1} R_{2}} m \cos (\phi)}{\gamma R_{2}+\gamma R_{1} m^{2}-F_{1}^{2} R_{2}-F_{2}^{2} R_{1} m^{2}-2 F_{1} F_{2} \sqrt{R_{1} R_{2}} m \cos (\phi)}
$$

The maximum of (17) occurs in which

$$
\begin{aligned}
& \frac{\partial \eta}{\partial m}=0, \\
& \frac{\partial \eta}{\partial \phi}=0,
\end{aligned}
$$

and

$$
\frac{\partial \eta}{\partial \beta}=0
$$

are simultaneously satisfied. After some algebraic arrangements, the solutions are obtained as

$$
\begin{gathered}
\beta=\sqrt{1+F_{1}^{2}+F_{2}^{2}}=\beta_{o p t}, \\
m=\left|\frac{V_{2}}{V_{1}}\right|=\left|\frac{F_{2} \sqrt{R_{2}}}{F_{1} \sqrt{R_{1}}}\right|=\left|\frac{k_{20} \sqrt{L_{2}}}{k_{10} \sqrt{L_{1}}}\right|=\left|\frac{M_{2}}{M_{1}}\right|,
\end{gathered}
$$

and

$$
\phi=\left\{\begin{array}{ll}
0^{\circ} & \left(F_{1} F_{2}>0\right) \\
-180^{\circ} & \left(F_{1} F_{2}<0\right)
\end{array} .\right.
$$

Consequently, (22) and (23) can be combined into one equation given by

$$
m=\frac{V_{2}}{V_{1}}=\frac{F_{2} \sqrt{R_{2}}}{F_{1} \sqrt{R_{1}}}=\frac{k_{20} \sqrt{L_{2}}}{k_{10} \sqrt{L_{1}}}=\frac{M_{2}}{M_{1}} .
$$

As indicated, $m$ is a real number. Maximum efficiency is mostly not obtained with the use of $V_{1}=V_{2}$, which is usually applied in practice. When inserting these solutions into (17), the maximum efficiency is expressed as 


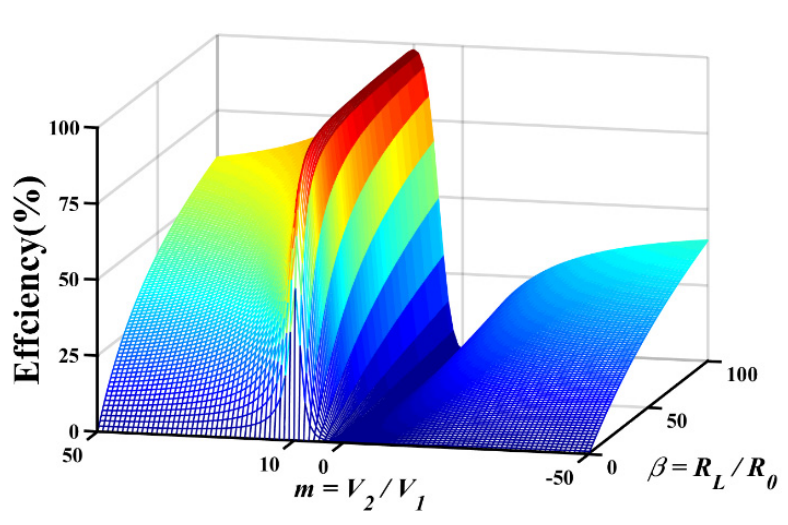

Fig. 3. Power transfer efficiency as a function of $m$ and $\beta$.

$$
\eta_{\max }=\frac{\frac{\beta_{o p t}}{\gamma_{o p t}}\left(F_{1} \sqrt{R_{2}}+\frac{F_{2}^{2} \sqrt{R_{2}}}{F_{1}}\right)^{2}}{\gamma_{\text {opt }}\left(R_{2}+\left(\frac{F_{2} \sqrt{R_{2}}}{F_{1}}\right)^{2}\right)-\left(F_{1} \sqrt{R_{2}}+\frac{F_{2}^{2} \sqrt{R_{2}}}{F_{1}}\right)^{2}}
$$

where

$$
\gamma_{o p t}=1+F_{1}^{2}+F_{2}^{2}+\beta_{o p t}=1+F_{1}^{2}+F_{2}^{2}+\sqrt{1+F_{1}^{2}+F_{2}^{2}} .
$$

After further algebraic simplification, (24) results in

$$
\eta_{\max }=\frac{\sqrt{1+F_{1}^{2}+F_{2}^{2}}-1}{\sqrt{1+F_{1}^{2}+F_{2}^{2}}+1}=\frac{F_{1}^{2}+F_{2}^{2}}{\left[1+\sqrt{1+F_{1}^{2}+F_{2}^{2}}\right]^{2}}=\frac{F^{2}}{\left[1+\sqrt{1+F^{2}}\right]^{2}}
$$

where $F\left(=\sqrt{F_{1}^{2}+F_{2}^{2}}\right)$ is defined as the figure of merit for a MISO system $(N=2)$. For a MISO system with $N$ transmitters, we define the system figure of merit as $F=\sqrt{\sum_{n=1}^{N} F_{n}^{2}}$. Clearly, (27) has the same form as (1) with $b=1$. However, note that this is true only when the couplings between the Tx's are negligible.

In Fig. 3, we plot the efficiency (17) as a function of $m$ and $\beta$ for the case of $F_{1}=10, F_{2}=100$, and $R_{0}=R_{1}=R_{2}=0.01 \Omega$. Fig. 3 shows that $m$ and $\beta$ must have specific values to achieve maximum efficiency. When the voltage ratio $m=V_{2} / V_{1}=10$ and the normalized load impedance $\beta=\sqrt{1+F_{1}^{2}+F_{2}^{2}}=100.1$, as shown in (21) and (24), respectively, efficiency is shown to become maximum (92\%). The common practice of using the same Tx voltages $\left(V_{2}=V_{1}\right)$ in a MISO may result in a much lower efficiency than the achievable maximum.

\section{CIRCUIT AND ELECTROMAGNETIC SIMULATIONS}

To validate the theory in Section II, circuit and EM simulations are performed for the case of $r_{1}=r_{2}=6 \mathrm{~cm}, r_{0}=5 \mathrm{~cm}, d=$ $10 \mathrm{~cm}, c=0 \mathrm{~cm}$, and $g=20 \mathrm{~cm}$ in Fig. 4. The Tx and Rx loops are made of copper rings with a radius of $1 \mathrm{~mm}$. In this case, $R_{1}$

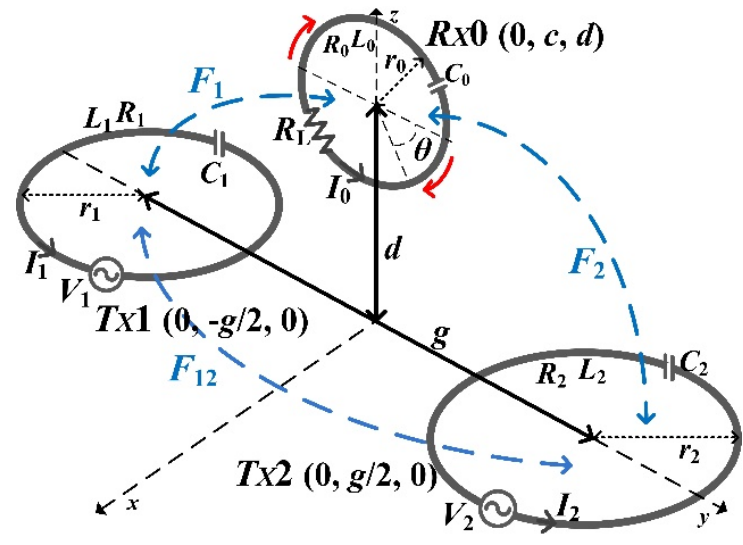

(a)

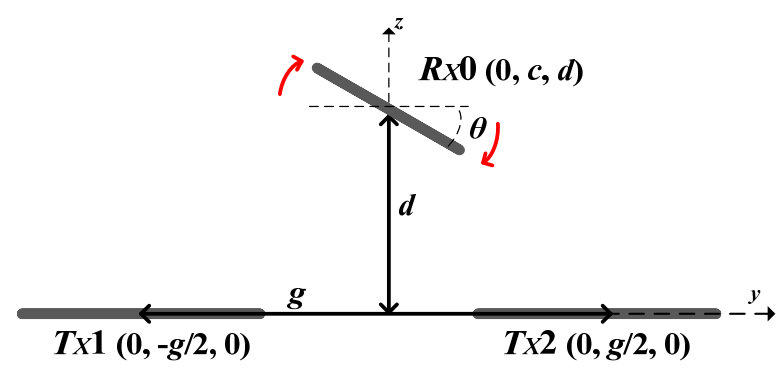

(b)

Fig. 4. MISO $(N=2)$ WPT system with an angular misalignment angle $\theta$ ( $g=$ distance between the center of two Tx's, $d=$ vertical distance between the origin and $\mathrm{Rx} 0$ ): (a) overall view and (b) front view.

$=R_{2}=0.0408 \Omega, R_{0}=0.034 \Omega, L_{1}=L_{2}=0.335 \mu \mathrm{H}$, and $L_{0}=$ $0.266 \mu \mathrm{H}$. We choose $C_{1}=C_{2}=1.65 \mathrm{nF}$ and $C_{0}=2.07 \mathrm{nF}$ for resonance at $6.78 \mathrm{MHz}$. The quality factors $Q_{1}$ (or $Q_{2}$ ) and $Q_{0}$ are 348.6 and 334.2 , respectively. The coupling coefficient $\left(k_{21}\right)$ [11] between the two Tx's is -0.0064 . The minus sign implies that the magnetic flux generated from one Tx loop crosses the other in an opposite direction. Thus, the figure of merit $F_{12}=$ -2.23. The GA in MATLAB (optimization toolbox) is applied to find the optimum magnitude and phase of $V_{2} / V_{1}$ and the $\mathrm{Rx}$ load resistance $\left(R_{\mathrm{L}}\right)$ through which the efficiency reaches a maximum. EM simulation was performed by ANSYS HFSS using copper loops and lumped C components for resonance. From the EM simulation, we obtain the scattering matrix and coupling coefficients between all copper loops.

Fig. 5(a) presents the figures of merit $\left(F_{1}, F_{2}\right)$ and optimum load resistances using the GA and Eq. (21) as a function of the misalignment angle $\theta$ at $0^{\circ}-90^{\circ}$. While $F_{1}\left(=k_{10} \sqrt{Q_{1} Q_{0}}\right)$ gradually increases from 2.9 to $5.3, F_{2}\left(=k_{20} \sqrt{Q_{2} Q_{0}}\right)$ decreases from 2.9 to -5.3 crossing zero at $\theta=30^{\circ}$. The negative $F_{2}$ comes from the downward magnetic flux on $\operatorname{Rx} 0$ when $\theta>30^{\circ}$. The magnitude of $F_{12}$ is shown to be smaller than that of $F_{1} . F_{2}$ changing sign from positive to negative at $\theta=30^{\circ}\left(F_{2}=0\right)$ can be understood by observing the direction of the magnetic flux. The optimum load resistance $\left(R_{L, o p t}\right)(21)$ is almost the same as 


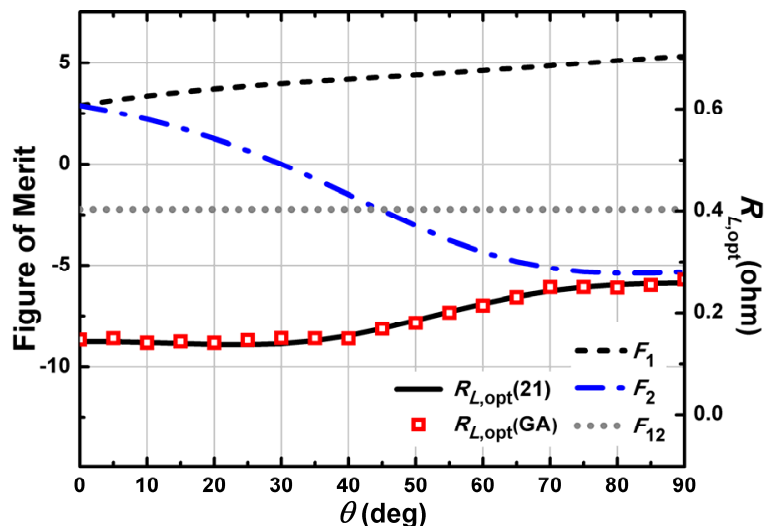

(a)

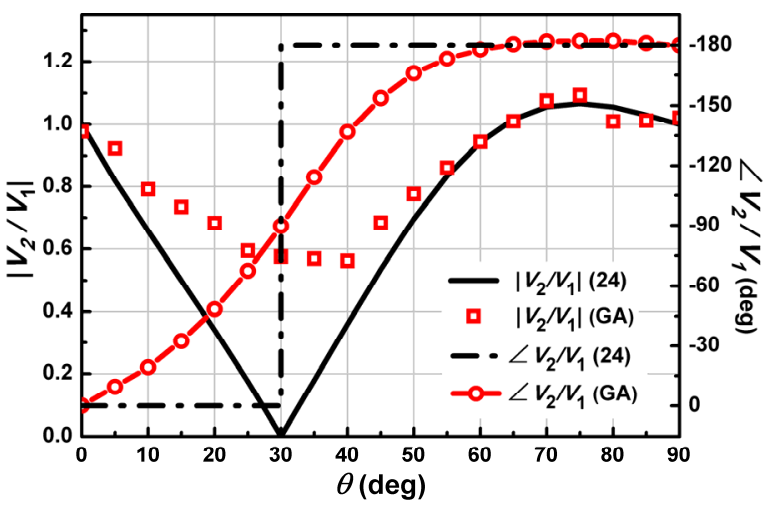

(b)

Fig. 5. MISO $(N=2)$ system parameters, optimized loads, and input voltage ratio $\left(V_{2} / V_{1}\right)$ as a function of the misalignment angle $\theta$ for the case of $r_{1}=r_{2}=6 \mathrm{~cm}, r_{0}=5 \mathrm{~cm}, d=10 \mathrm{~cm}, c$ $=0 \mathrm{~cm}$, and $\mathrm{g}=20 \mathrm{~cm}$ in Fig. 4 : (a) figures of merit $\left(F_{1}\right.$ and $F_{2}$ ) and optimum load, and (b) magnitude and phase of $m$ $\left(=V_{2} / V_{1}\right)$.

the result obtained from the GA even though $F_{12}(=-2.23)$ is not considered in (21).

Fig. 5(b) shows the optimized magnitude and phase of the voltage ratio $\left(m=V_{2} / V_{1}\right)$ as a function of the misalignment angle $\theta$ using the GA and (24). The magnitudes and phases of $V_{2} / V_{1}$ using the GA and (24) are in agreement except at around $\theta=30^{\circ}$. The discrepancies around $\theta=30^{\circ}$ comes from the fact that, while the assumption made for (24) is the smaller mutual coupling $\left(\left|F_{1}\right|>\left|F_{12}\right|\right)$ and $\left.\left|F_{2}\right|>\left|F_{12}\right|\right),\left|F_{2}\right|$ is much smaller than $\left|F_{12}\right|$ at around $\theta=30^{\circ}$.

In Fig. 6, the efficiencies based on the GA, Eqs. (21, 24, 8), Eq. (27), and EM-simulation are plotted as a function of the misalignment angle for the same system. The efficiencies for the SISO systems using only Tx1 or Tx2 are also included for comparison. The $\operatorname{MISO}(21,24,8)$ is calculated by inserting Eqs. $(21,24)$ into (8). The MISO $(27)$ and MISO (GA) are shown to be in excellent agreement. The EM-simulated efficiency MISO (EM) using $\left|V_{2} / V_{1}\right|$ (GA) and $\angle V_{2} / V_{1}$ (GA) in Fig. 5(b) also agrees with them. These efficiencies are shown to be higher than those of the SISO systems. The MISO $(21,24,8)$ is also

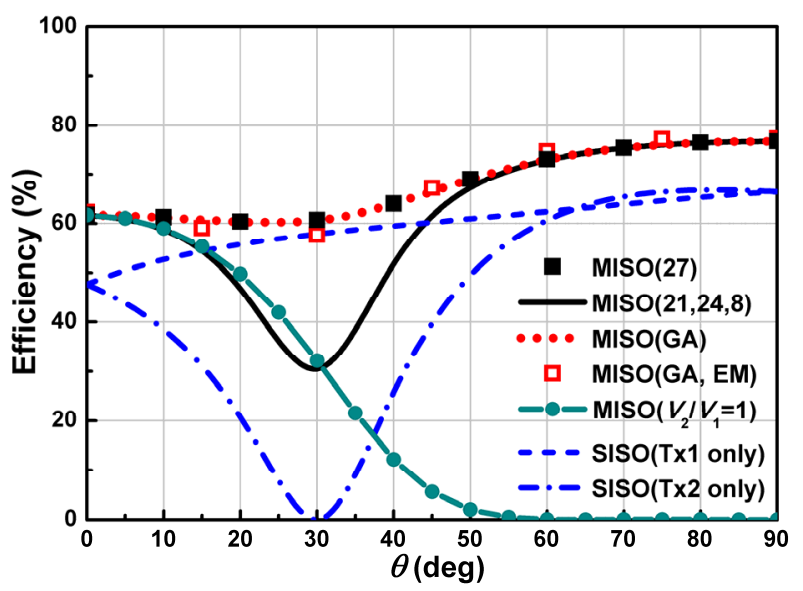

Fig. 6. MISO $(N=2)$ and SISO system transfer efficiencies as a function of the misalignment angle $\theta$.

shown to be higher than the SISO in which $\left|F_{12}\right|(=2.23)$ is roughly smaller than the system figure of merit $F$. As $\left|F_{12}\right|>F$, near $\theta=30^{\circ}$, we need to optimize the system parameters considering the mutual couplings. However, the evaluation of the maximum MISO efficiency is shown to have been exactly conducted with (27) not depending on the information of mutual coupling. The common practice of using the same $\mathrm{Tx}$ voltages $\left(V_{2}=V_{1}\right)$ is against the solution (22) and leads to much lower efficiencies than the achievable maximum as shown by MISO $\left(V_{2} / V_{1}=1\right)$ in Fig. 6.

\section{CONCLUSION}

The misalignment problems have been shown to be always solved by MISO through the numerical optimization of $\mathrm{Tx}$ excitations (magnitudes and phases). They have been analytically obtained and provided in this study when the mutual inductances are relatively small. Further research is needed to fabricate the proposed MISO system and validate the theory with experimental results.

This research was supported by the MSIT (Ministry of Science and ICT), Korea, under the Information Technology Research Center support program (No. IITP-2019-2016-000291) supervised by the Institute for Information \& Communications Technology Promotion (IITP).

\section{REFERENCES}

[1] A. Kurs, A. Karalis, R. Moffatt, J. D. Joannopoulos, P. Fisher, and M. Soljacic, "Wireless power transfer via strongly coupled magnetic resonance," Science, vol. 317, no. 5834, pp. 83-86, 2007.

[2] D. Ahn and S. Hong, "Effect of coupling between multiple 
transmitters or multiple receivers on wireless power transfer," IEEE Transactions on Industrial Electronics, vol. 60, no. 7, pp. 2602-2613, 2013.

[3] X. Mou, O. Groling, and H. Sun, "Energy-efficient and adaptive design for wireless power transfer in electric vehicles," IEEE Transactions on Industrial Electronics, vol. 64, no. 9, pp. 7250-7260, 2017.

[4] H. Hu, S. Yao, K. Bao, and S. V. Georgakopoulos, "Misalignment insensitive WPT with conformal SCMR systems," in Proceedings of 2015 IEEE International Symposium on Antennas and Propagation \& USNC/URSI National Radio Science Meeting, Vancouver, Canada, 2015, pp. 117-118.

[5] J. P. K. Sampath, A. Alphones, and D. M. Vilathgamuwa, "Figure of merit for the optimization of wireless power transfer system against misalignment tolerance," IEEE Transactions on Power Electronics, vol. 32, no. 6, pp. 4359-4369, 2016.

[6] S. Goguri, R. Mudumbai, and A. Kruger, "Optimizing wireless power transfer with multiple transmitters," in Proceedings of 2017 51st Annual Conference on Information Sciences and Systems (CISS), Baltimore, MD, 2017, pp. 1-5.

[7] Q. Zhu, M. Su, Y. Sun, W. Tang, and A. P. Hu, "Field

Hyeongwook Lee

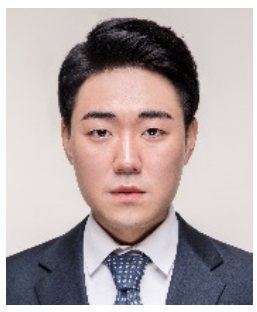

received the B.S. in electronic engineering from Kyung Hee University, Yongin, Korea, in 2019. He is currently working toward the M.S. degree in electronic engineering in Kyung Hee University. His research interests are microwave antenna, passive devices, and wireless power transmission. orientation based on current amplitude and phase angle control for wireless power transfer," IEEE Transactions on Industrial Electronics, vol. 65, no. 6, pp. 4758-4770, 2018.

[8] T. Mei, F. Liu, C. Jiang, X. Chen, and R. M. Kennel, "Magnetic-field-model based analysis of two-phase magnetically coupled resonant wireless power transfer system," in Proceedings of 2018 IEEE Applied Power Electronics Conference and Exposition (APEC), San Antonio, TX, 2018, pp. 1092-1097.

[9] P. Kong and H. Ku, "Efficiency optimising scheme for wireless power transfer system with two transmitters," Electronics Letters, vol. 52, no. 4, pp. 310-312, 2016.

[10] H, Li, K. Wang, L. Huang, J. Li, and X. Yang, "Coil structure optimization method for improving coupling coefficient of wireless power transfer," in Proceedings of 2015 IEEE Applied Power Electronics Conference and Exposition (APEC), Charlotte, NC, 2015, pp. 2518-2521.

[11] G. Kim and B. Lee, "Alternative expressions for mutual inductance and coupling coefficient applied in wireless power transfer," Journal of Electromagnetic Engineering and Science, vol. 16, no. 2, pp. 112-118, 2016.

Seunghyun Boo

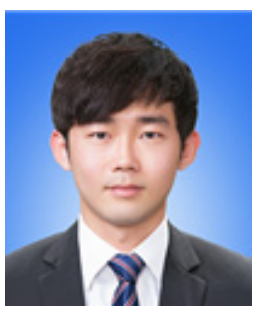

received his B.S. and M.S degrees in electronics engineering from Kyung Hee University, Yongin, Korea, in 2017 and 2019, respectively. His research fields include microwave antennas, passive devices, and wireless power transmission. 


\section{Gunyoung Kim}

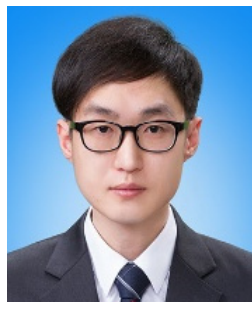

received a B.S. in Radio Communication Engineering from Kyung Hee University, Yongin, Korea in 2010, an M.S. in Electronics and Radio Engineering from Kyung Hee University in 2012, and a Ph.D. in Electronics Engineering from Kyung Hee University in 2018. Currently, he is a post-doctor in the College of Electronics and Information at Kyung Hee University. His fields of research include microwave antennas, passive devices, wireless power transmission, and metamaterials.

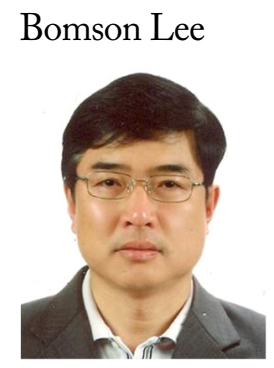

received the B.S. degree in Electrical Engineering from Seoul National University, Seoul, Korea, in 1982. From 1982 to 1988, he was with the Hyundai Engineering Company Ltd., Seoul, Korea. He received the M.S. and Ph.D. degrees in Electrical Engineering from the University of Nebraska, Lincoln, NE, USA, in 1991 and 1995, respectively. In 1995, he joined the faculty at Kyung Hee University, where he is currently a professor in the Department of Electronics and Radio Engineering. From 2007 to 2008, he was the chair of the technical group for microwave and radio wave propagation in the Korea Institute of Electromagnetic Engineering \& Science (KIEES). In 2010, he was an editor-in-chief of the Journal of the Korean Institute of Electromagnetic Engineering and Science. In 2018, he served as the president of KIEES. His research activities include microwave antennas, RF identification (RFID) tags, microwave passive devices, wireless power transfer, and metamaterials. 